\section{Structure of a New System of CO Bands}

Is a previous note ${ }^{1}$ the discovery of a new system of red-degraded $\mathrm{CO}$ bands, placed just before the heads of the violet-degraded Third Positive system, was reported. Inspection and measurement of highdispersion spectrograms now show that the new bands have a structure appropriate to a ${ }^{3} \Sigma \rightarrow^{3} \Pi$ bandsystem, the lower state being the $a^{3} \Pi$ state, which is the usual final state of the CO triplet systems. The upper ${ }^{3} \Sigma$ levels of the new bands lie about $83705 \mathrm{~cm}^{-1}$ and $85885 \mathrm{~cm}^{-1}$ above the $\mathrm{CO}$ ground state; they have observable spin-splittings and rotational constants $B^{\prime}=0.7$ to $0.8 \mathrm{~cm}^{-1}$. The system may tenta. tively be designated as $f^{3} \Sigma \rightarrow a^{3} \Pi$. It may be considered, however, as probably due to transitions from the higher vibrational levels of $a^{\prime 3} \Sigma$ to the lower vibrational levels of $a^{3} \Pi$. For, within the heights of the initial levels $f^{3} \Sigma$ of the new bands occur also the initial levels $b^{3} \Sigma$ of the Third Positive bands, and the latter are strongly perturbed, as has already been established ${ }^{2}$, by vibrational levels of the $a^{\prime 3} \Sigma$ state, for which we have predicted term values and rotational constants of the magnitude now observed for the new bands.

Rotational analysis and details will be published shortly elsewhere.

Physical Institute,

R. SснміD.

L. GERö.

Royal Hungarian University,

Budapest. July 31.

1 Schmid, R., and Gerö, L., Naturwiss., 25, 90 (1937).

${ }^{2}$ Schmid, R., and Gerö, L., Z. Phys., 105, 36 (1937) (1937).

L-Emission Bands of Zinc, Copper, Nickel and Cobalt

I HAve studied the intensity of the $L$-emission bands of metals from $\mathrm{Co}(27)$ to $\mathrm{Zn}(30)$. The apparatus used was a vacuum spectrograph using a bent crystal of mica, and the source of the radiation was an X-ray tube.

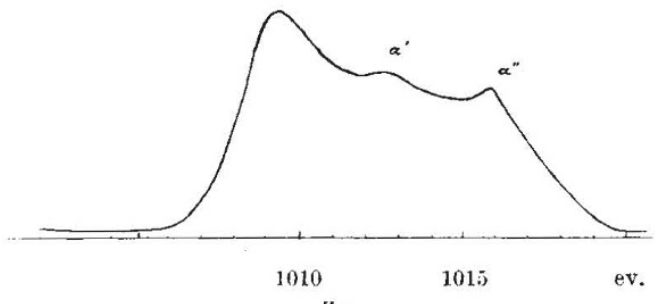

$\mathrm{Zn}$

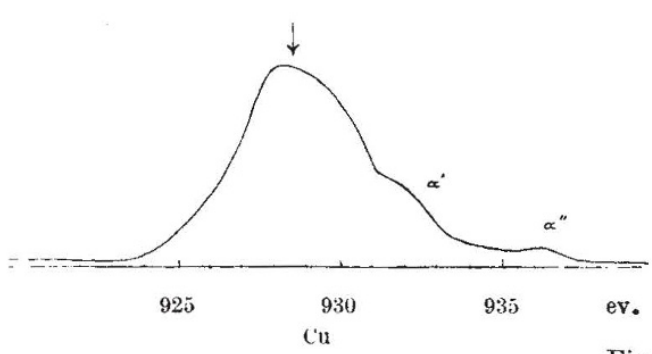

Fig. 1

To obtain the actual intensity distribution of the emitted radiation, the plate was calibrated by a method already described ${ }^{1}$. The curves giving the intensity of the $L_{a}$ bands against energy (in electronvolts) are shown in Fig. 1 .
It is well known that the $L$-emission bands are emitted when $M_{\text {Iv }}$, v-electrons make transitions into

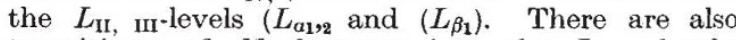
transitions of $N_{\mathrm{I}}$-electrons into the $L_{\mathrm{II}}$, II-levels. For these metals the $N_{I^{-}}$or $4 s$-electrons are the conduction electrons, and occupy levels which overlap in energy those of the $M_{\mathrm{IV}}, \mathrm{v}^{-}$or $3 d$-electrons. ${ }^{2}$ Thus the observed bands represent transitions of both $3 d$ - and $4 s$ - electrons. But for copper and zinc, unfortunately, the short wave-length ends of the bands, which are probably due to the $4 s$-electrons, are completely masked by satellites, which are strongly enhanced by the reorganization of the atom on account of the Auger effect ${ }^{3}, L_{\mathrm{I}}-L_{\mathrm{III}}$.

The position of the short wave-length edge of the $L_{a}$-bands may be estimated from the $L$-absorption spectra, and is given by the wave-length of the slope of the absorption curve near the minimum absorption. For copper, we can deduce from the absorption curve of Sandström ${ }^{4}$ that the edge of the $L_{a}$-emission band is given by the arrow marked on Fig. 1. From the comparison of the $L$-emission and absorption bands it is obvious that the width of the $L_{a}$-band is about $5 \mathrm{ev}$.

Université, Liége.

J. FARINEAU. Aug. 3.

${ }^{1}$ Comptes rendus, 204, 1242.

${ }^{2}$ See Mott and Jones, "The Theory of the Properties of Metals and Alloys" (Oxford, 1936), p. 191.

${ }^{3}$ See Coster and Kronig, Physica, 2, 13 (1935).

- Sandström, A., Thesis, Uppsala (1935).

$M$-Emission Bands of Zinc, Copper and Nickel

WE have had the opportunity of seeing $\mathrm{Dr}$. Farineau's letter before it was sent to press. We have recently observed the $M$-emission bands of nickel, copper and zine metals, and it seems worth while to add a note, since the two sets of results, especially taken together, allow one to draw certain conclusions about the structure of the $3 d$ - and $4 s$ (conduction)-electron bands in these metals.

Our plates, taken with a concave grating spectro. graph in the region $130-210 \mathrm{~A}$. and with an X-ray

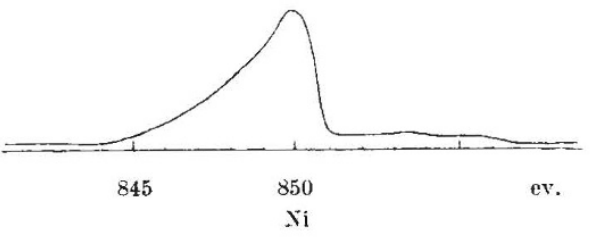
tube run at 3,000 v. as source, give results which, on a scale of energy, are al. most identical with Farineau's $L$ - em is sion bands. This is good evidence that, apart from transition probability factors, the curves represent features of the density. functions of the combined $3 d$. conduction. levels of the metals. We also obtain for zinc and copper the peaks marked $\alpha^{\prime}$ and $\alpha^{\prime \prime}$ by him. But, contrary to his conclusion, when we compare the wave-lengths of points on the $M$-emission bands with the wave-lengths of the $M$-absorption edges which we have determined ${ }^{1}$, we 